problems serious enough to interfere with anaesthesia increases in a markedly non-linear fashion with increasing age. $\mathrm{Up}$ to age 45 adults (and children) who are generally well are good candidates for day-case admission and assessment on admission will rarely result in postponement or cancellation of the operation except in the presence of acute respiratory or other infections. From 45 to 65 , however, the prevalence of chronic cardiovascular, respiratory, and metabolic disease starts to become significant and minimal outpatient assessment for day-case surgery should probably include the taking of a full medical history, the measurement of the resting blood pressure, and auscultation of the heart and chest. Patients over 65 should rarely, I believe, be booked for day-case surgery without full outpatient assessment of fitness for anaesthesia, usually including electrocardiography and chest $x$-ray.

Self-administered questionnaires would seem particularly suited to use as an aid to historytaking in the preoperative assessment of patients undergoing routine surgery provided that the surgeon or anaesthetist who sees the patient preoperatively discusses his responses with him, and provided also (at least for the over-45s) that preoperative assessment is done at a time which allows for correction of any significant abnormality found before operation.

It cannot be over-emphasised that a surgeon who books a patient for day-case surgery is certifying, by implication, that the patient is fit for anaesthesia, and that the admission of a patient, booked for operation on the same day, who is not fit for the anaesthetic results is a very considerable waste of time and money for all concerned.

Sheffield

P R FlETCHER

\section{Rapid serological diagnosis of outbreaks} of influenza

SIR,-The current activity of both type A and $B$ influenza viruses is associated with the usual crop of outbreaks of febrile respiratory illness in schools, hospitals, institutions, and similar groups of persons. It may be helpful to remind clinicians that the specific diagnosis of such outbreaks can usually be established by routine serological tests within 24 hours of the laboratory receiving one collection of blood samples from patients with different durations of illness, including both the shortest intervals possible (for example, patients who sickened the same day) and those of over one week. This method is technically simpler than virus isolation or immunofluorescent demonstration of viral antigen in respiratory secretions, which provide alternative diagnostic manoeuvres within the first week of an outbreak.

In 1961 influenza A was serologically proved as the cause of an outbreak among geriatric patients, of whom $20 \%$ were ill, several had lower respiratory complications, and one died. Complement-fixing (CF) antibody titres to influenza $A$ were 8 or less in 11 whose illness began within the previous week but were 16 , 64 , and 256 respectively in three ill for a week or longer. ${ }^{1}$ Confirmatory rising titres were subsequently demonstrated in second sera. Psittacosis from a ward pet was suspected in a similar episode in January 1975 but no psittacosis CF antibodies were found (thus exonerating the bird), whereas titres ranging from 16 to 1024 + for influenza A were found in five men whose illnesses began 2-4 weeks previously.

From an outbreak at a naval air station in 1963 six sera were taken from men with illnesses of 1-3 days' duration and six from men whose illnesses began 8-27 days previously. All tests in the first group were negative, whereas three in the second group had significant influenza A CF antibody titres. This immediate diagnosis was later confirmed by isolation of A2 influenza virus and demonstration of significantly rising antibody titres in all five men from whom second blood samples were received.

From a similar outbreak in a military camp in March 197021 blood samples were collected-16 taken in the first week of illness (influenza B CF titres insignificant in 14, $128+$ in 2: geometric mean titre of group 16) and five in the second week of illness (all 5 titres $128+$ : geometric mean titre 256 to influenza B). ${ }^{2}$

In principle this method is applicable to outbreaks of infection other than influenza. Differences of titres of groups with different durations of illness have similar diagnostic significance to differences in titres of paired acute and convalescent sera from the same patient.

University Department of

Norman R GRIST Infectious Diseases,

Glasgow

Grist, N R, Kerr, J, and Isaacs, B, British Medical Fournal, 1961, 2, 431

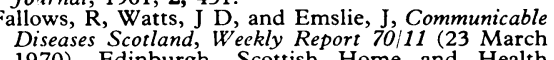

1970). Edin

\section{Dispersal of biliary calculi by irrigation}

SIR,-May I be allowed to counsel against the procedure advocated by $\mathrm{Mr} \mathrm{S} \mathrm{J}$ A Powis (7 February, p 340)?

There is only one sure method of guaranteeing that the common bile duct is clear and this is by direct inspection with a choledochoscope. No urologist would regard a bladder examination as complete without a cystoscopy, and the same applies with the bile ducts. When this is done it is possible to inspect the sphincter of Oddi and if this is clear then it is not necessary to drain the common bile duct in any but the most exceptional cases.

For this procedure gentle irrigation is employed, and this is safe; but irrigation strong enough to flush out the duct is hazardous and the only complication we have had from choledochoscopy has been when this was done and the patient developed septicaemia.

General Hospital

G T WATTS

Birmingham

\section{Preventing animal diseases}

SIR,-The veterinary profession welcomes the call for increased medical/veterinary liaison expressed in your leading article (14 February, p 355).

We look to the medical profession to support our efforts to bring the supervision of the hygiene of food products of animal origin under veterinary control. The present system of meat inspection, based on the search for a near-extinct disease, tuberculosis, largely ignoring the hygiene of the lairages and the producers' premises, and without bacteriological control, means that salmonella infections, the spread of which is encouraged by intensification of animal production, can pass the whole of the inspection system unobserved and, dare we say, unlooked for. Poultry is deemed not to exist.

EEC directives may force Britain to a standard of meat hygiene under veterinary supervision which has been the norm in most developed countries for many years, although sections of the Environmental Health Officers' Association are fighting a not wholly unsuccessful rearguard action to retain an outdated system. The Verdon-Smith Committee Report in $1964^{1}$ and the recent Swann Committee Report $^{2}$ both unequivocally recommended a centralised, veterinary controlled meat hygiene system.

Can we ask for BMA support in taking British meat hygiene screaming and struggling into the twentieth century?

J Allcock Chairman, Public Relations Committee,
British Veterinary Association

London W1 into Fatstock and Carcase

Committee of Inquiry into Fatstock and Carcase
Meat Marketing and Distribution, Report, Cmnd 2282. London, HMSO, 1964 . Committee of Inquiry into the Veterinary Profession,
Report, Vol I, Cmnd 6143. London, HMSO, 1975.

SIR,-In response to your leading article (14 February, $p$ 355) and Sir James Howie's letter ( $p$ 393) we wish to present evidence for the value of monitoring for brucellosis.

From 1967 attempts to eradicate brucellosis in cattle were made by means of the voluntary brucellosis schemes. Figures from the local Department of Agriculture and Fisheries for Scotland showed that by November 1973, when compulsory eradication was started in Ayrshire, $92 \%$ of all breeding herds in the county were members of the voluntary schemes, $72 \%$ of these member herds being accredited. By December $197598.6 \%$ of all dairy herds and $95.8 \%$ of beef herds were accredited. A total of 5267 reactors and 7096 contact animals had been slaughtered at a cost of well over f2m in compensation.

Specimens of cow milk sent to our laboratory for brucella tests increased from 493 in 1971 to 1324 in 1975 . All milk samples were investigated by the milk ring test (MRT) for the presence of agglutinins to Brucella abortus. The percentage of milks showing a positive MRT decreased progressively each year from $83(16.8 \%)$ in 1971 to $1(0.07 \%)$ in 1975 . The total of 228 milks with positive MRT results during 1971-5 were also tested by whey agglutination, considered to be uninfluenced by previous vaccination, and by culture for brucella organisms. Positive whey agglutination results were obtained with 177 of the milks and positive cultures with 45 . The proportion of MRT-positive milks which were also positive by the two other tests fell progressively each year from $31 \%$ in 1971 to nil in 1975.

During 1971-5 human brucellosis was diagnosed in 53 patients in Ayrshire (46 males and 7 females). Thirty-four of these had serological findings suggesting chronic or past infection diagnosed for the first time. The remaining 19 patients were diagnosed as "acute" brucellosis on the basis of clinical symptoms and/or serological titres $\geqslant 1600$ 